\title{
Haemodynamic effects of oral aminorex and amphetamine in unanaesthetized beagle dogs
}

\author{
JAMESA. WILL' and GERALD E. BISGARD \\ Cardiovascular Research Laboratory, Department of Medicine, University of Wisconsin Medical School and \\ Department of Veterinary Science, College of Agriculture and Life Sciences, University of Wisconsin, \\ Madison, Wisconsin 53706
}

\begin{abstract}
The haemodynamic effects of four daily oral doses of $1.5 \mathrm{mg} / \mathrm{kg}$ of aminorex (as fumarate) were measured in unanaesthetized beagle dogs. A similar study was performed in another group of related animals using d-amphetamine sulphate $(1 \mathrm{mg} / \mathrm{kg})$. Both agents produced similar sympathomimetic effects consisting of transient rises in systemic and pulmonary arterial blood pressures over an eight-hour period after administration of either drug. Heart rate remained unchanged in the dogs receiving aminorex whereas it decreased in those receiving amphetamine.

There was no cumulative or residual haemodynamic effect of either drug. We therefore conclude that neither aminorex nor amphetamine readily produces sustained pulmonary hypertension in beagle dogs.
\end{abstract}

Aminorex $($ Menocil(R)) is an appetite suppressant which possesses certain features of chemical structure common to both amphetamine and epinephrine. The pharmacological activity of aminorex and amphetamine as appetite suppressants has been attributed to their ability to release endogenous catecholamines (Peters and Gourzis, 1970).

An increase in incidence of primary pulmonary hypertension has been found in patients treated for obesity with aminorex (Gurtner et al., 1968). Because of this circumstantial evidence animal experiments have been carried out in order to test the effect of aminorex and other appetite suppressants on pulmonary haemodynamics.

It is quite well established that chlorphenteramine, phenteramine, aminorex, and amphetamine administered intravenously in acute experiments cause a transient elevation of systemic arterial and pulmonary arterial pressures in dogs (Peters and Gourzis, 1970; Leuschner, Otto, and Wagener, 1970; Brunner and Stepanek, 1971; Aalund and Boeck, 1970). In calves with highly reactive pulmonary vascular beds, acute intravenous injection of aminorex or amphetamine caused an increase in systemic arterial pressure but had no effect on pulmonary artery pressure (Grover and ByrneQuinn, 1970).

More prolonged administration of aminorex has

1Requests for reprints to: Dr. James A. Will, Cardiovascular Research Laboratory, 420 North Charter, Room 523, Madison Wisconsin 53706 been attempted in animals in order to simulate the clinical situation in patients receiving the drug. Administration of aminorex to rats for 302 days and to beagle dogs for 140 days failed to produce $\frac{\mathbb{Q}}{\varnothing}$ pulmonary hypertension as assessed by measure- $\stackrel{\vec{F}}{\vec{F}}$ ments or right ventricular weights at necropsy and $\frac{0}{3}$ histological examination of the pulmonary vascular $\vec{J}$ system (Kay, Smith, and Heath, 1970). In another study in 54 beagle dogs by Leuschner et al. (1970), there was no increase in pulmonary artery or femoral artery pressures after 6 to 13 weeks' treatment with aminorex, amphetamine, or other related $\underset{\sigma}{\times}$ compounds. These workers also reported no 3 . increase in heart weight and no morphological or histological signs of damage in either the hearts or lungs of these dogs. Engelhardt and Hort (1970)응 reported an increase in pulmonary artery pressure in rats given high doses of aminorex for periods of 29 to 123 days, but no evidence of right ventricular hypertrophy or changes in pulmonary vessels were $N$ found in any animal. They concluded that factorsin other than aminorex were probably responsible for reproducible anatomical alterations in the pul- $\omega$ monary circulation. In work reported by Peters and Gourzis (1970) there were no cardiopulmonarye lesions in dogs studied after 13 months and in ratse studied after 18 months of aminorex treatment.

Using twice the average normal human dose of aminorex $(0.25 \mathrm{mg} / \mathrm{kg} /$ day $)$, Grover and ByrneQuinn (1970) found no increase in mean pulmonary artery pressure (PAM) during a one-month study 
in calves with highly reactive pulmonary vascular beds. They also noted no changes in another group of calves receiving amphetamine.

In contrast to these negative findings, Brunner and Stepanek (1971) found that the oral administration of $1.5 \mathrm{mg} / \mathrm{kg}$ of aminorex (as fumarate) daily for four days caused an increase in resting pulmonary artery pressures on the third and fourth days. This response persisted in a few dogs up to 10 days after the last dose.

The serious implications of this conflicting report by Brunner and Stepanek (1971) and our own interest in animal models of pulmonary hypertension led us to attempt to confirm the results of their study. We therefore studied the direct effects of aminorex and amphetamine on pulmonary and systemic haemodynamics using a protocol similar to that of these investigators.

\section{MATERIALS AND METHODS}

AMINOREX STUDIES Thin-walled Teflon catheters were surgically implanted in 12 adult beagle dogs aged 8 months to 3 years and weighing from 7 to $13 \mathrm{~kg}$. The Teflon catheters had an inside diameter of approximately 0.038 in and an outside diameter of 0.062 in. The dogs, six male and six female, were anaesthetized with sodium pentothal and maintained with methoxyfluorane. Under aseptic conditions catheters were passed into the carotid artery and jugular vein and directed to the descending aorta and pulmonary artery respectively under image intensified fluoroscopy. During fluoroscopy the catheters were filled with sodium and meglumine diatrizoates (Hypaque(R) M 90\%). The free ends of the catheters were passed underneath the skin to a point on the back of the neck, tied in place, and fitted with threeway disposable stopcocks. On the day following surgery, all dogs were placed in a restraint rack in sternal recumbency and the catheters were checked for patency and proper placement. Studies began four days after catheter placement. At the end of each day the catheters were filled with heparin solution $(1,000$ units $/ \mathrm{ml}$ ) and each dog was given $30,000 \mathrm{u} / \mathrm{kg}$ procaine penicillin $G$ intramuscularly. The rack in which the dogs were studied was specially made to accommodate eight dogs simultaneously without discomfort.

The protocol was as follows: the strain gauges were levelled for each dog for each measurement, using the point of the shoulder as an indication of the right atrial level. Each morning (hour 1) of days 1 to 4 inclusive, resting pulmonary artery and aortic pressures and heart rate were measured on all dogs when catheters were patent. Following these initial resting measurements, each dog was given a capsule containing $1.5 \mathrm{mg} / \mathrm{kg}$ of aminorex (as fumarate) based on the initial weight of the animal taken three days before the start of the experiment. On days 5 and 12 , the same protocol was followed except that no drug was administered. On days 1,4 , and 12 , indicator dilution curves using indocyanine green dye to determine cardiac output and pulmonary and systemic resistances were inscribed. These measurements were done at hours 1, 2, and 9 for these days. Statham P-23 Db strain gauges were used for the measurement of all pressures, and recordings were made on an Electronics for Medicine oscillographic recorder. Calibrations were made before and at the end of each day's procedures with the exception of the calibration for indicator dilution curves which was done at the end of the trial using pooled blood.

AMPHETAMINE STUDIES The same protocol as above was carried out in a second group of beagles from the same kennel. This group received d-amphetamine sulphate $(1 \mathrm{mg} / \mathrm{kg})$. Seven dogs were prepared; however, due to catheter problems, only three or four dogs were available throughout the studies. These dogs were trained for one week before catheterization and for days 1 and 2 after catheters were in place. Studies began on day 3 . The drug was also administered for the first four days of the study. Measurements were made hourly for four hours on the fifth day (day 7); no drug was given on that day.

\section{RESULTS}

AMINOREX STUDIES The unpaired $t$ test described by Steel and Torrie (1960) was used to analyse the data for groups which had unequal variances and numbers of animals. Comparisons were made

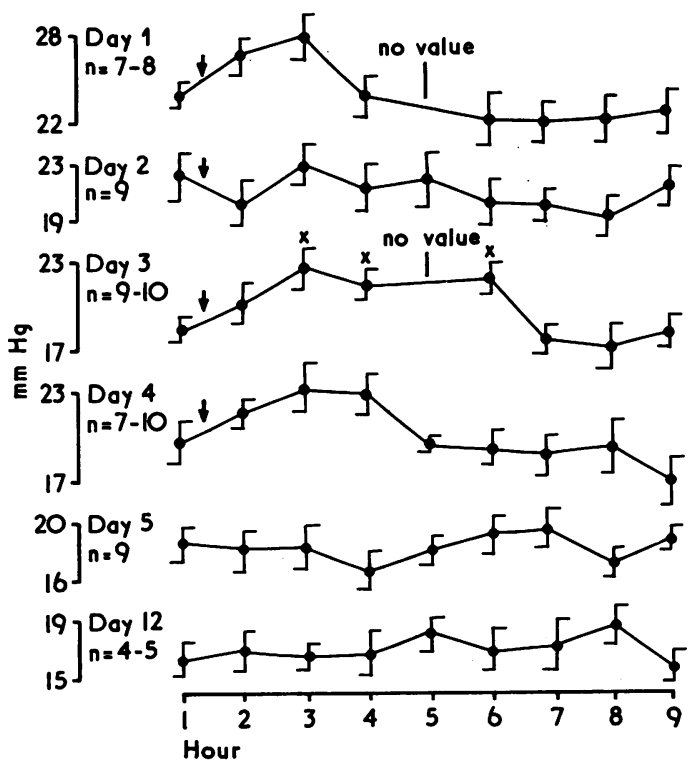

FIG. 1. Average pulmonary artery mean pressures for the control period (hour 1) and each succeeding hour of every experimental day. $X$ indicates a significant difference from hour $1(P<0.05)$; the arrows indicate when drug was administered. Vertical bars $=$ standard error of the mean (S.E.M.). 
between hour 1 of each day (control period) and each succeeding hour on that day. While the drug appeared to increase PAM (peaking two hours after drug administration), significant increases were found only on hours 3,4 , and 5 of day 3 $(P<0.05$, Fig. 1). No changes occurred in pulmonary artery pressures on days 5 and 12 when no drug was administered.

In order to test for a potential cumulative effect, as reported by Brunner and Stepanek (1971), comparisons were made between hour 1 of day 1 and hour 1 of every other day. No cumulative effect was noted; there was instead a slight decrease in PAM which was significantly decreased on day 3

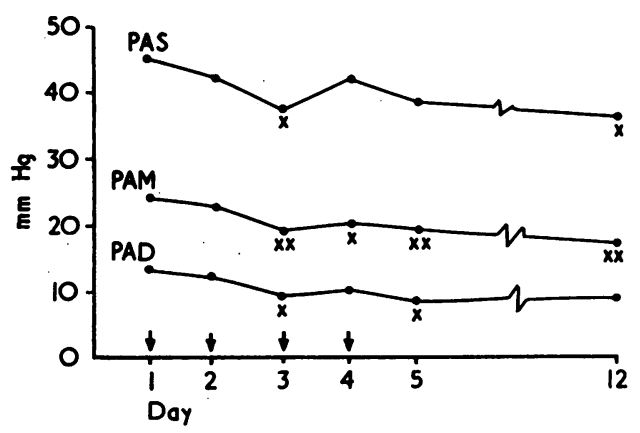

FIG. 2. Average pulmonary artery pressures from the control periods (hour 1) of each experimental day. PAS, $P A D$, and $P A M$ signify systolic, diastolic, and mean pulmonary artery pressures respectively. $X$ indicates $a$ significant difference from hour 1 , day $1(P<0.05) ; X X$ indicates a very significant difference $(P<0.01)$.

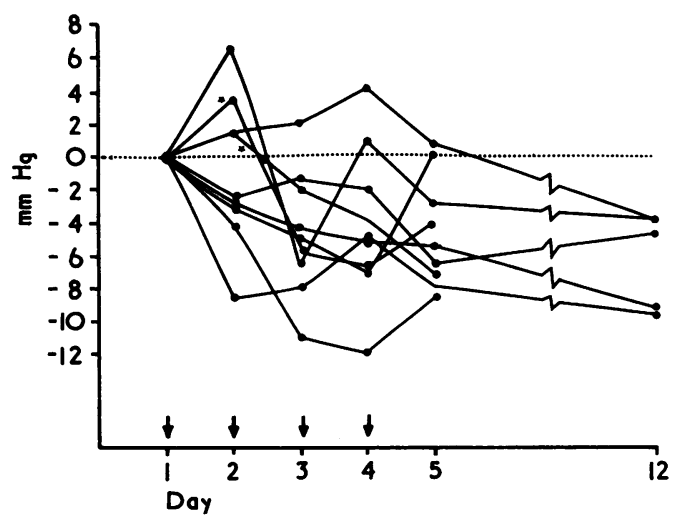

FIG. 3. Deviations of control (hour 1) mean pulmonary artery pressures from each dog on each experimental day from the control period of day 1. Stars $(\star)$ signify dogs 11 and 12 in which the pressures were not recorded on day 1. The mean of the seven other dogs was used as the day 1 control pressure. The arrows indicate days on which the drug was administered.

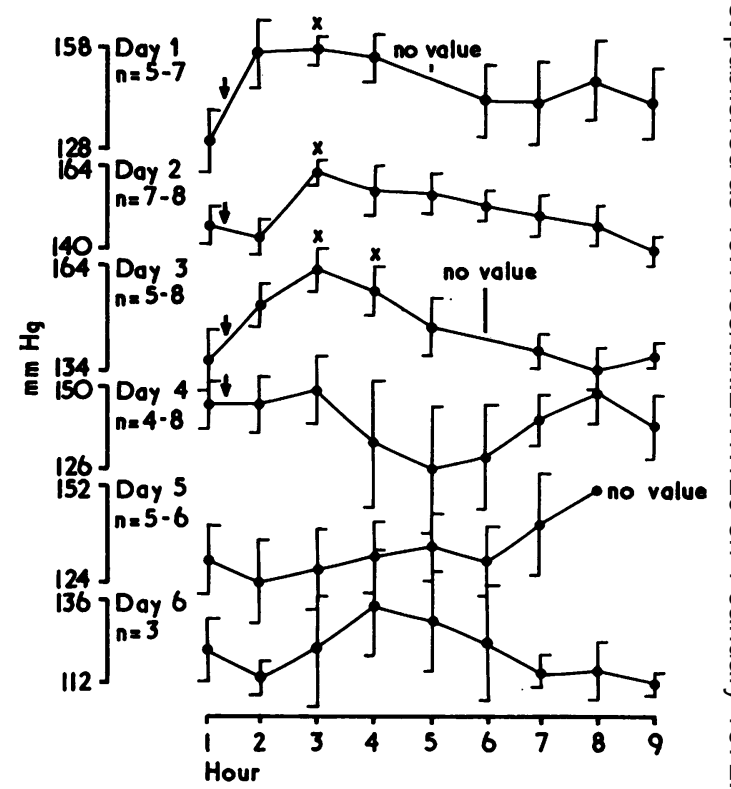

FIG. 4. Average aortic mean pressures from the control period (hour 1) and each succeeding hour of every experimental day. $X$ indicates a significant difference from hour 1 $(P<0.05)$; vertical bars equal S.E.M.

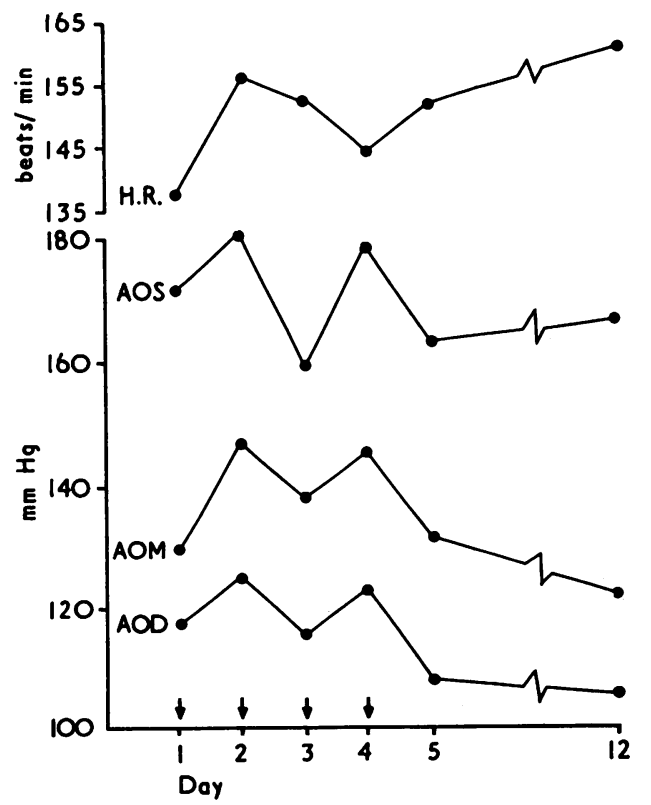

FIG. 5. Mean heart rate and average aortic pressures from control period (hour 1) of each experimental day. AOS, $A O D$, and $A O M$ signify systolic, diastolic, and mean $\frac{\rho}{\Phi}$ aortic pressures respectively. Arrows indicate days on $\_$ which the drug was administered. 
$(\mathrm{P}<0.01)$, day $4(\mathrm{P}<0.05)$, and days 5 and 12 $(\mathbf{P}<0.01)$. Systolic and diastolic pulmonary pressures followed a similar pattern (Figs 2 and 3 ).

Significant increases in aortic pressures were noted only during hour 3 of days 1,2 , and 3 and the fourth hour of day 3 (Figs 4 and 5).

No effects of aminorex on heart rate were found with the exception that the initial control heart rate on day 2 was significantly higher than in the succeeding hours. This was also the case on day 12 when the initial value was compared to the final value of the day (Fig. 5).

No important changes resulting from the administration of aminorex were found in cardiac output, stroke volume, total peripheral resistance or total pulmonary resistance (Fig. 6).

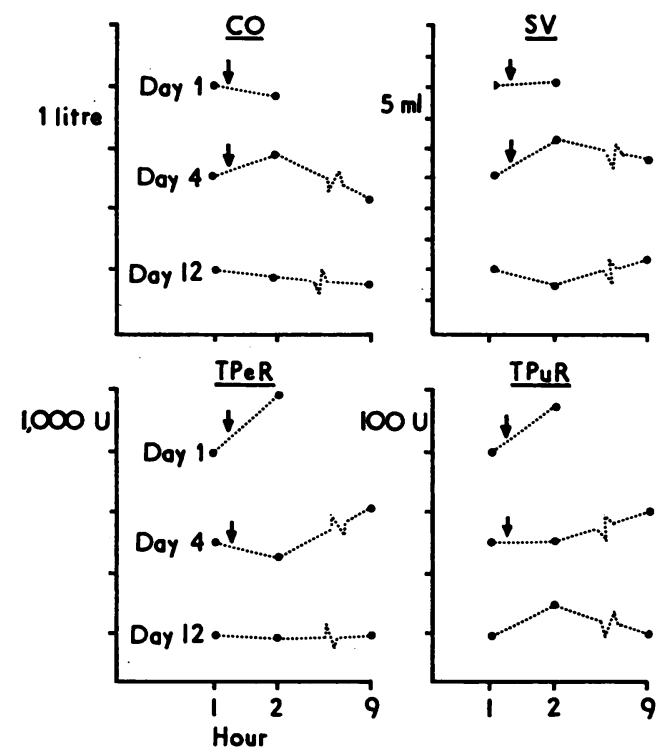

FIG. 6. Cardiac output (CO), stroke volume (SV), total peripheral resistance (TPeR), and total pulmonary resistance (TPuR) for the hours when measured. Arrows indicate when drug was administered.

AMPHETAMINE STUDIES While no statistically significant haemodynamic changes occurred in the amphetamine study definite trends were apparent. Heart rates tended to decrease with time following the administration of the drug. Aortic and pulmonary arterial pressures tended to rise, reaching a peak at about hour 4 and returning toward control levels by the end of each day. Cardiac output remained essentially the same while stroke volume increased. Total peripheral resistance tended to increase while total pulmonary resistance stayed about the same (Figs 7 to 11).

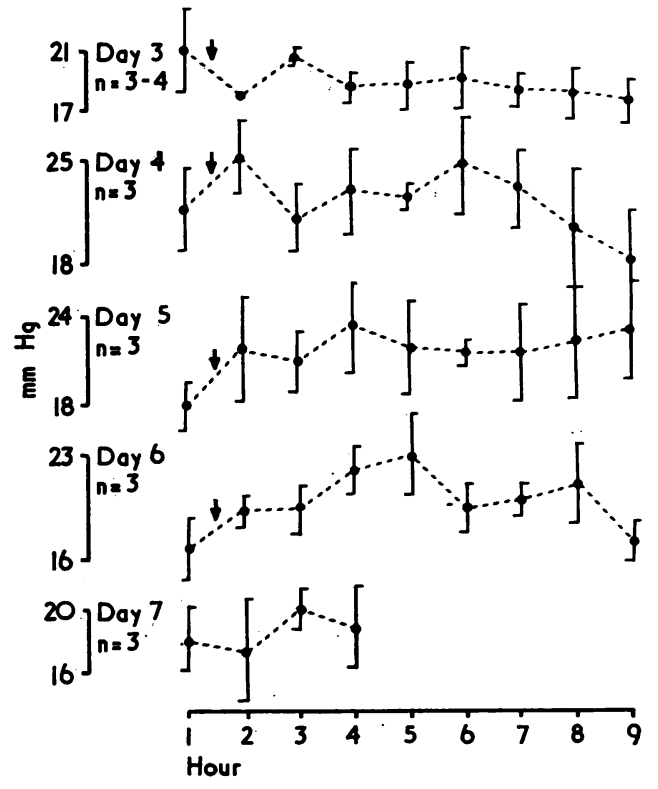

FIG. 7. Average pulmonary artery mean pressures from the control period (hour 1) and each succeeding hour of every experimental day; arrows indicate when drug was administered; vertical bars equal S.E.M.

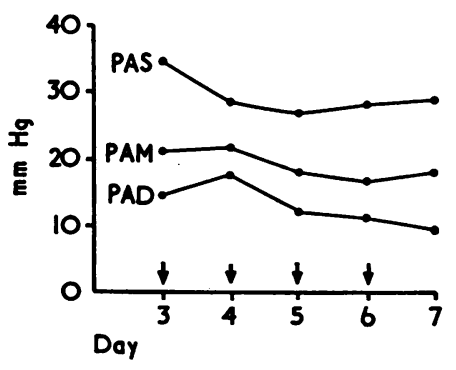

FIG. 8. Average pulmonary artery pressures from the control periods (hour 1) of each experimental day. PAS, $P A D$, and $P A M$ signify systolic, diastolic, and mean pulmonary artery pressures respectively. Arrows indicate days on which drug was administered.

\section{DISCUSSION}

We attempted to use the same protocol as that of Brunner and Stepanek (1971) in our study; however, we used closely related pure bred beagle dogs from a single supplier in order to maintain a greater degree of uniformity in the groups. There were no significant differences attributable to sex or age.

It was immediately apparent that these dogs had resting mean pulmonary artery pressures higher 


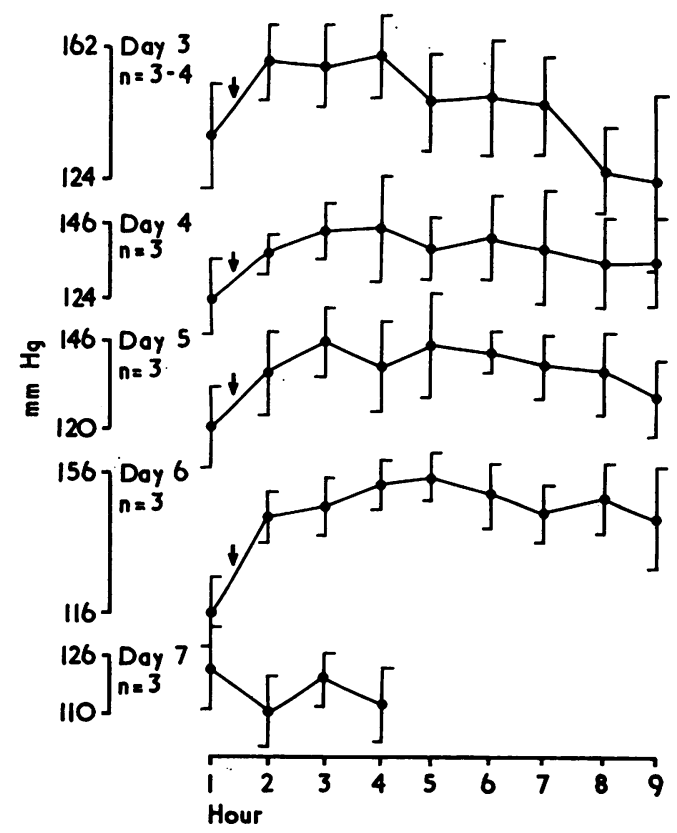

FIG. 9. Average aortic mean pressures from the control period (hour 1) and each succeeding hour of every experimental day. Arrows indicate when drug was administered; vertical bars equal S.E.M.

than one would expect from excitement or from mild exercise. Even though the amphetamine dogs were trained for a longer period of time, the first day control PAMs were not significantly lower than in the aminorex dogs. Because of the potential effects of training we examined five of these beagle dogs two months after the end of the experiments described in this paper. At this time the dogs were considered to be highly trained and accustomed to being handled. Pulmonary arterial pressures and heart rates were recorded in these dogs while they were unanaesthetized and at rest in sternal recumbency. The average PAM was $15.2 \pm 2.7 \mathrm{mmHg}$ (mean \pm S.D.). The heart rate was $120 \cdot 4 \pm 15 \cdot 1$ beats/minute. These PAM values were significantly lower than those recorded on day 1 hour 1 (control pressures) in both the aminorex and amphetamine studies. However, they were not significantly different from post-control pressures (hour 1 , days 5 and 12 in the aminorex study; hour 1 , day 7 in the amphetamine study). This implies that there was indeed a significant training effect. It should also be pointed out that beagle dogs have been reported to have higher resting pulmonary artery pressures $(18.4 \pm 1.1 \mathrm{mmHg}$; Aalund and Boeck, 1970) than mongrel dogs $(8-16 \mathrm{mmHg}$;

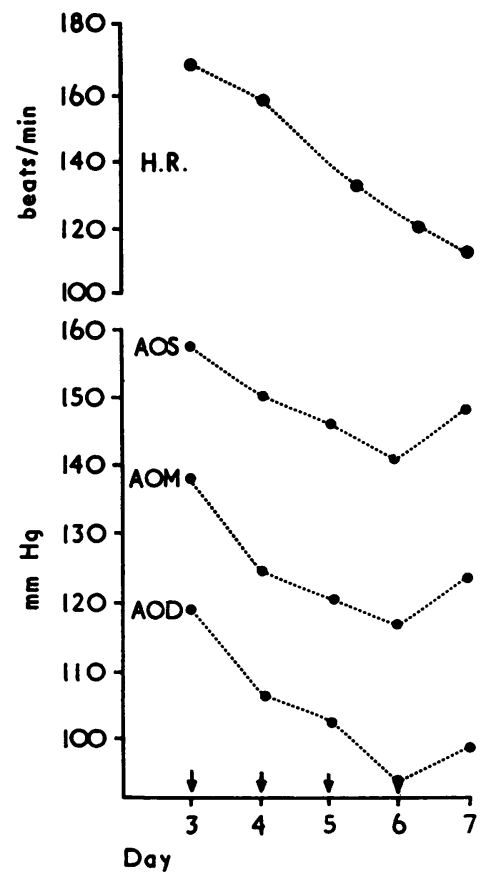

FIG. 10. Mean heart rate and average aortic pressures from the control period (hour 1) of each experimental day. $A O S, A O D$, and $A O M$ signify systolic, diastolic, and mean aortic pressures respectively. Arrows indicate days $\stackrel{\circ}{\circ}$ on which drug was administered.

Yarns and Tashjian, 1967). Even though the resting pulmonary artery pressures of beagles appear high compared to mongrel dogs, average pressure rises of $4 \mathrm{mmHg}$ or more sometimes occurred in $\times$ the beagles in our aminorex study. This further implies that pulmonary vasoactivity is still present, particularly since cardiac output did not appear to increase.

Dirofilariasis (heart-worm infestation) is a po- $\frac{?}{?}$ tential cause of pulmonary hypertension, but the dogs used in the present study were raised in a colony for experimental purposes and certified as $\mathcal{N}$ being free of the disease; moreover, if the disease $N$ were present, it would tend to potentiate any vaso- N active effects of a drug. Chronic pulmonary hyper- $\omega$ tension due to the hypoxia of high altitude is known to produce greater reactivity of the pul-

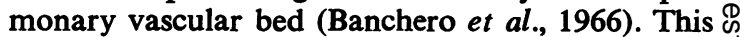
suggests that dogs with a mild degree of resting pulmonary hypertension might be more susceptible to the effects of a pulmonary vasoconstricting $\underset{\mathbb{D}}{\mathbb{D}}$ agent. Furthermore, the effects of acute hypoxia $\stackrel{?}{\mathbb{P}}$ in our five trained dogs was tested by anaesthe- $\varrho$ tizing them with $20-25 \mathrm{mg} / \mathrm{kg}$ of pentobarbital 


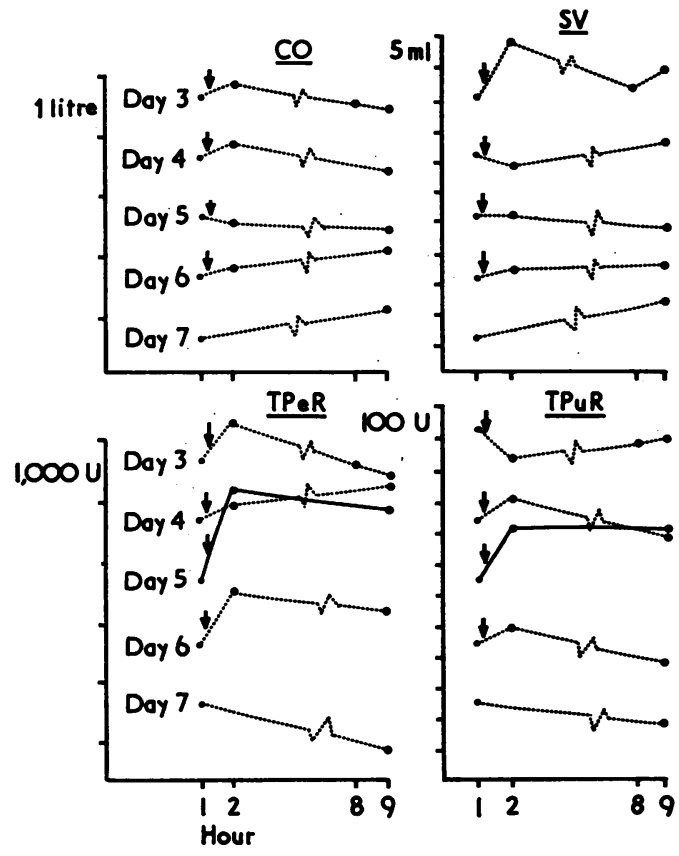

FIG. 11. Cardiac output (CO), stroke volume (SV), total peripheral resistance $(T P e R)$, and total pulmonary resistance (TPuR) for the hours when measured. Arrows indicate when drug was administered.

sodium and administering $40 \% \quad \mathrm{O}_{2}+60 \% \quad \mathrm{~N}_{2}$ followed by $8 \% \quad \mathrm{O}_{2}+92 \% \quad \mathrm{~N}_{2}$. The PAM was increased markedly from $14 \cdot 2 \pm 2 \cdot 8$ when the $\mathrm{PaO}_{2}$ was only $140 \mathrm{mmHg}$ to $23.0 \pm 4.9(\mathrm{P}<0.01)$ during hypoxia $\left(\mathrm{PaO}_{2}-2130 \mathrm{mmHg}\right)$. This demonstrates that the dogs used in our experiments had ample cardiovascular responsiveness.

Following the administration of aminorex, the mean pulmonary artery pressure did show a consistent increase, reaching a peak two hours after administration. The increase was significant only on day 3 ; however, this trend is in agreement with other previously cited acute experiments.

The significant difference between our work and that of Brunner and Stepanek (1971) is in the day to day changes in PAM. These workers reported an incremental increase in the control PAM beginning on the third day of drug administration and reaching a peak of approximately $5 \mathrm{mmHg}$ above the control level on the fourth day; this increase persisted for up to 10 days in two dogs. No cumulative or residual effect of the drug on pulmonary artery pressure was apparent from one day to the next in our dogs (Fig. 3); instead, there was a steady decrease in the average PAM of all dogs (Fig. 2). Only one dog had pressures consistently higher than control and this animal's PAM returned to the first day (control) level on the fifth day. If there was a residual drug effect it should have been present at this time. Eight days after the last dose of drug the mean pulmonary artery pressure was more than $7 \mathrm{mmHg}$ below control.

Another possible cause for differences in the results of our studies is the feeding pattern of the experimental animals. Our dogs consumed a normal amount of food because this was available overnight. The presence or absence of food in the digestive tract may have influenced the absorption and excretion of the drug in the experiments of other workers.

As previously indicated, Professor Brunner's dogs were large mongrels weighing 15 to $25 \mathrm{~kg}$, approximately twice the size of our beagles. This raises the question whether absolute dose is more important than the dose per unit of body weight.

The results we obtained in the amphetamine studies are in agreement with those of other workers (Aalund and Boeck, 1970; Brunner and Stepanek, 1971). No residential or cumulative haemodynamic effects were found.

Profound changes were noted in the behaviour and clinical signs following administration of aminorex to these dogs. Extreme nervousness, restlessness, hyperactivity, and pupillary dilatation were noted. It was interesting to note that the food was consumed by the next morning when offered ad libitum, verifying the fact that the duration of appetite suppression was less than 24 hours. Amphetamine-treated dogs did not show evidence of anorexia nor the other clinical signs displayed by those receiving aminorex, but it must be remembered that the comparative dosage of amphetamine was approximately one-half that of aminorex.

This investigation has demonstrated that neither aminorex nor amphetamine readily produces sustained pulmonary hypertension in beagle dogs. Thus, the beagle dog can be added to the list of animals in which the production of sustained pulmonary hypertension was not achieved by the administration of sympathomimetic appetite suppressants. However, it could be that susceptible animals do exist but have not been included among the limited number studied.

The authors wish to acknowledge the kind assistance of Dr. Tonny Ungerer, James Orr, Gordon Johnson, Rita Lesczynski, Betty Lange, and Mary Risberg.

Special thanks are also extended to Dr. George G. Rowe, Director of the Cardiovascular Research Laboratory, for helpful advice. 
This study was supported in part by the College of Agricultural and Life Sciences, University of Wisconsin, and by McNeil Laboratories Inc., Fort Washington, Pennsylvania.

Dr. Bisgard is supported by U.S.P.H.S. Special Fellowship No. 36984.

\section{REFERENCES}

Aalund, M., and Boeck, V. (1970). The acute effect of chlorphentermine, d-amphetamine and phentermine on the pulmonary and systemic arterial pressure in nonanesthetized dogs. Address at Symposium: Obesity, Circulation and Anorexigens, May 15-16, Buergenstock, Switzerland.

Banchero, N., Sime, F., Penaloza, D., Cruz, J., Gamboa, R., and Marticorena, E. (1966). Pulmonary pressure, cardiac output, and arterial oxygen saturation during exercise at high altitude and at sea level. Circulation, 33, 249.

Brunner, H., and Stepanek, J. (1971). Effects of aminorex on the pulmonary circulation of the dog. Proceedings of the European Snciety for the Study of Drug Toxicity $12,123$.

Engelhardt, R. and Hort, W. (1970). Cardiovascular effect of aminorex in rats after prolonged application. NaunynSchmiedebergs Arch. Pharmak., 266, 318.
Grover, R. F., and Byrne-Quinn, E. (1970). Aminorex and amphetamine: chronic effects of pulmonary and systemic hemodynamics in the calf. Address at Symposium: Obesity, Circulation and Anorexigens, May 15-16, 1970, Buergenstock, Switzerland.

Gurtner, H. P., Gertsch, M., Salzmann, C., Scherrer, M., ำ Stucki, P., and Wyss, F. (1968). Is there an increase incs the primary vascular forms of chronic cor pulmonale? Schweiz. med. Wschr., 98, 1579 and 1695.

Kay, J. M., Smith, P. H., and Heath, D. A. (1970). A quanti- $\vec{\omega}$ tative pathological study of the heart and pulmonary arteries of rats and dogs after prolonged feeding with aminorex fumarate (Menocil). Address at Symposium: $\vec{x}$ Obesity, Circulation and Anorexigens, May 15-16, 1970, N Buergenstock, Switzerland.

Leuschner, F., Otto, H., and Wagener, H. H. (1970). The tolerance of aminorex and some other compounds during prolonged administration to beagle dogs. Naunyn-Schmiedebergs Arch. Pharmak., 266, 391.

Peters, L., and Gourzis, J. T. (1970). Pharmacological, toxico- logical and clinical observations with aminorex in the United States. Address at Symposium: Obesity, Circula-J tion and Anorexigens, May 15-16, 1970, Buergenstock, Switzerland.

Steel, R. G. D., and Torrie, J. H. (1960). Principles and Procedures of Statistics, p. 81. McGraw-Hill, New York.

Yarns, D. A., and Tashjian, R. J. (1967). Cardiopulmonary? values in normal and heartworm-infected dogs. Amer. J. vet. Res., 28, 1461. 